\title{
TOPOLOGY OPTIMIZATION IN THE CASE OF DIFFERENT BOUNDARY CONDITIONS BY AN ITERATIVE METHOD
}

\author{
JÁNOS LÓGÓ \\ Department of Structural Mechanics, Budapest University of Technology and Economics \\ H-1111 Budapest, Mûegyetem rkp. 3, Hungary \\ logo@ep-mech.me.bme.hu
}

[Received: December 12, 2005]

\begin{abstract}
Optimal design with thousands of variables is a great challenge in engineering calculations. In this paper an iteration based topology design technique is introduced for the optimization of linearly elastic continuum type structures under single parametric static loading and different displacement boundary conditions. The support optimization is discussed briefly, as well. The investigated problem is utilized by minimization of the weight of the structure subjected to displacement constraints. The numerical procedure is based on an iterative formula which is formed by the use of the Kuhn-Tucker condition of the Lagrangian function of the constraint mathematical programming problem. The application is illustrated by numerical examples.
\end{abstract}

Mathematical Subject Classification: 74P15

Keywords: mathematical programming, topology optimization, optimal design

\section{Introduction}

The past century has produced impressive improvements in power and efficiency of mathematical programming techniques as applied to general structural design problem. These methods with their generality do not allow significant number of design variables and number of constraints. This tends to restrict their usefulness to problems with from tens to a few hundreds of design variables. Attempts to apply numerical search procedures to resize problem have failed due to the fact of the large number of design variables involved or the huge computational expenses. The special approaches which have solved such problems successfully are known from the literature as optimality criteria methods.

Recently the topology optimization is one of the most "popular" topic in the expanding field of optimal design. A great number of papers indicates the importance of the topic $[1,3-5,7,9,11,23,25,27,28]$. The popularity comes one part from the needs of the industry (car, airplane, etc.) and other part from the complexity of the problem which is a great challenging for the researchers. In present stage the field of topology optimization can be divided into two subfields: optimal design of skeletal structures (trusses, grids, etc.,) deals with the simultaneous optimization of the member sizes 
and geometry (coordinates of joints). This field is named layout optimization. The other subfield is called generalized shape optimization which deals with the simultaneous optimization of the topology and shape of the internal boundaries of the continuum type structures made of composite or porous materials. This subfield is based on the use on the Finite Element method and in the fundamental problem class the optimal structure is "built up" from empty (no material) and solid (filled with given isotropic material) elements.

The history of the topology optimization is approximately 100 years old. At the first 80 years a skeletal structure (usually a truss) was the object of the optimization problem. Exactly one hundred years ago Michell [13] used the full stress design (FSD) criteria on statically determinate structures to obtain the minimum weight structure. In 1957 Barta [2] extended this method to statically indeterminate structures and found that FSD did not converge to the minimum weight design. The other important intuitive method was the uniform strain energy density design. Formal methods of the calculus of variation, applied by Taylor [29], Prager and his co-workers [see e.g. $15,16,17]$ to specific distributed parameter problems, showed great promise, suggesting that implementation to discretized (e.g., FEM) models could lead to a new class of general purpose optimization methods. The first discretized optimality criteria methods were based on strain energy distributions characterizing optimum structural designs for stress constraints. As Prager and Taylor [15] showed, if the work of the applied loads is limited as an equality constraint, the optimum structure with uniform material properties has uniform energy density distribution.

The origin of the classical optimality criteria method (COC) is dated back into the 70-es. Turning attention to certain stiffness constraints theoretically valid optimality criteria were derived for skeletal structures with displacement constraints employing classical Lagrangian multiplier methods of mathematical programming optimization. To satisfy the optimality criteria an algorithm (an iterative formula) was proposed by Berke [6] based on the attitude that this formula theoretically correctly guaranteeing at least a local optimum when satisfied. Berke also suggested that the design variables and constraints should be separated into a passive and an active sets. In 1991 Kaliszky and Lógó [10] presented a new type of application for the optimal design of dynamically loaded reinforced concrete frames under displacement and deformation constraint. The solution based on Berke's optimality criteria method with multiple constraints and a penalty parameter was used in the iterative formulation. The finite element based topology optimization was first explored in 1973 by Rossow and Taylor [18] and later Cheng and Olhoff [8]. In 1988 a new generation of problem formulation were created by Bendsoe and Kikuchi [3] using homogenization. A detailed description of continuum-type optimality criteria method was reported in Rozvany's books $[20,23]$ and several reports $[21,22,25,30]$. Their work opened a new "road" in research named topology optimization, but the common thing is that all of them use the basic idea of the classical optimality criteria.

It can be seen that it was enough a half decade to develop a new direction in field of structural optimization. At the beginning of 90-es the topology optimization method was split into two main parts: one part is based on the SIMP type method (Solid 
Isotropic Microstructures with Penalty) the other part is used homogenization. Allaire and Khons' work [1, 23] gives a clear description of the homogenization technique.

In the following an iterative technique (which is named SIMP method) and the connected numerical examples will be discussed in detail. The standard FEM computer program with quadrilateral membrane and truss elements is applied in the numerical calculation. The object of the design (so-called ground structure) is a rectangular disk with given loading (one parametric static) and support conditions (fix or/and elastic bars) . The material is linearly elastic. The design variables are the thickness or/and cross-sectional are of the finite elements. To obtain the correct optimal topology some filtering method has to be applied to avoid the so-called "checker-board pattern".

\section{Optimization problem}

2.1. Problem statement. For illustration purposes an engineering design problem is investigated. Let's given a design space (space means a volume which has to fill with structural elements), where the structure is constructed by truss and membrane elements. By the use of the FEM (Finite Element Method) definitions, let's consider the simple case of

- a 2D structure consisting of disk' ground elements $(g=1, \ldots, G)$ which must have a constant thickness $\left(t_{g}\right)$ of either $t_{g}=t_{\min }=0$ or $t_{g}=t_{\max }=1$, such that each ground elements $(g)$ contains one or several sub-elements $(e=$ $\left.1, \ldots, E_{s}\right)$, whose stiffness coefficients are linear homogeneous functions of the ground element thickness $t_{g}$, (Practically it means that the meshing consists of two parts, a primary and a secondary one.)

- The truss elements are given by their length and cross-sectional area. The above "thickness" normalization is used for the cross-sectional areas, as well. (The cross-sectional area is multiplied by $t_{g}$.)

- A single static loading with given boundary conditions and

- displacement constraints $(d=1, \ldots, D)$.

Using the above-normalized determination, the structure can easily be transformed to problems with a different prescribed maximum thickness $t_{g}=t_{\max }$. Due to the linear relations this is done by multiplying all loads by $t_{\text {max }}$, whilst stresses, strains and displacements do not alter their value.

2.1.1. The applied finite element technique. The design domain is discretized in the usual way. (Figure 1.) As it was mentioned 4 nodes disk elements (Figure 2) and $2 \mathrm{D}$ truss elements are used. In that way numerically the two types of element can be handled easily.

The nodes are interconnected by disk or/and bar elements. The applied shape functions of the disk's element are given as follows: $N_{1}(\xi, \eta)=\frac{1}{4}(1-\xi)(1-\eta)$; $N_{2}(\xi, \eta)=\frac{1}{4}(1+\xi)(1-\eta) ; N_{3}(\xi, \eta)=\frac{1}{4}(1+\xi)(1+\eta) ; N_{4}(\xi, \eta)=\frac{1}{4}(1-\xi)(1+\eta)$. The displacement boundary conditions are given in form of fix supports, elastic springs 


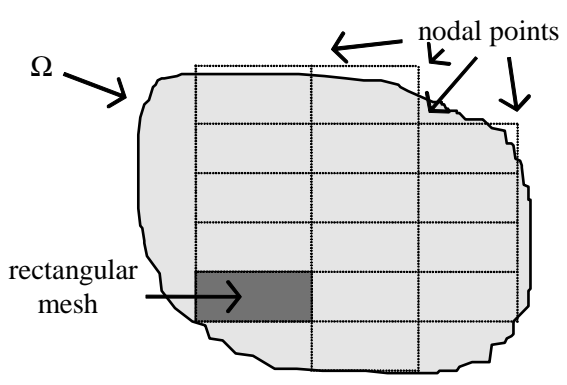

Figure 1. Discretization of the design domain

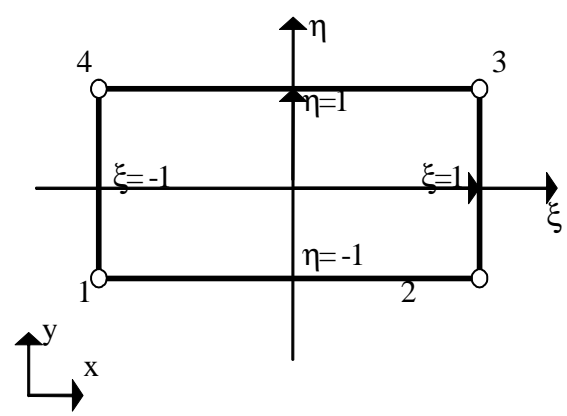

Figure 2. Local coordinate system and basic disk element

and elastic bars, respectively. The system stiffness matrix can be constructed by applying the assembling rule for iso-parametric elements.

2.1.2. Mathematical programming formulation. The structure is constructed by $\mathrm{G}$ piece of ground elements, where $G=N$ Disk $+N B a r$. Here NDisk is the number of the ground disk elements and $N B a r$ is the number of the bar elements.

The weight $(\mathrm{W})$ of the structure is given by

$$
W=\sum_{i d=1}^{n d i s c} \gamma_{i d} A_{i d} t_{i d}+\sum_{i b=1}^{n b a r} \gamma_{i b} A_{i b} \ell_{i b} t_{i b} .
$$

Here $\gamma_{i d}$ and $\gamma_{i b}$ the specific weight of the disk and bar elements, respectively. $A_{i d}$ is the area of the $i d$-th disk element, $A_{i b}$ is cross-section area and $\ell_{i b}$ the length of the $i b$-th bar element. If an artificial variable $A_{g}$ is introduced (in case of disk it means the area of the element $A_{g}=A_{i d}$, while in case of bar elements it has the following meaning: $\left.A_{g}=A_{i b} \ell_{i b}\right)$ the weight calculation can be expressed by the following compact form:

$$
W=\sum_{g=1}^{G} \gamma_{g} A_{g} t_{g},
$$

where $\gamma_{g}$ is the specific weight of the ground element g.

The displacement constraint can be expressed as

$$
\hat{\mathbf{u}}_{d}^{T} \mathbf{K u}-\Delta_{d} \leqslant 0 ;(d=1, \ldots, D)
$$

where $\hat{\mathbf{u}}_{d}$ is the virtual nodal displacement vector caused by the virtual loads (e.g. "unit dummy loads") associated with the displacement constraints, $\mathbf{K}$ is the system stiffness matrix, $\mathbf{u}$ is the nodal displacement vector associated with the real load and $\Delta_{d}$ is the prescribed limit on the displacement value in constraint $d$. 
The side constraints can be stated as

$$
\begin{aligned}
-t_{g}+t_{\min } \leqslant 0 ; & (\text { for } g=1, \ldots, G), \\
t_{g}-t_{\max } \leqslant 0 ; & (\text { for } g=1, \ldots, G) .
\end{aligned}
$$

In order to suppress the intermediate thicknesses, the weight calculation formulation is replaced by $\tilde{W}=\sum_{g=1}^{G} \gamma_{g} A_{g} t_{g}^{\frac{1}{p}}$ (where $p$ is the penalty parameter and $p \geqslant 1$ ). This gives the correct weight value for $t_{g}=0$ and $t_{g}=1$. The use of the penalty parameter has similar effect in the later formulations as it was the role of the penalty parameter in the classical optimality criteria method.

The optimization problem is to minimize the penalized weight of the structure which is subjected to a given single displacement and side constraints.

$$
\begin{aligned}
\tilde{W}=\sum_{g=1}^{G} \gamma_{g} A_{g} t_{g}^{\frac{1}{p}}= & \min ! \\
& \text { subject to }\left\{\begin{array}{r}
\mathbf{u}^{T} \mathbf{K} \mathbf{u}-\Delta \leqslant 0 ; \\
-t_{g}+t_{\min } \leqslant 0 ; \\
t_{g}-t_{\max } \leqslant 0 ;
\end{array} \quad(\text { for } g=1, \ldots, G),\right.
\end{aligned}
$$

In equation (4) the nodal displacement vector $\mathbf{u}$ associated with the real load $\mathbf{P}$ is calculated from the linear system of equations $\mathbf{K} \mathbf{u}=\mathbf{P}$.

2.1.3. Lagrange function. Using the Lagrange multipliers $v, \alpha_{g}, \beta_{g}$ and slack variables $h_{1}, h_{2 g}, h_{3 g}$ for the constraints in problem (4), the following Lagrange function can be formed:

$$
\begin{aligned}
& £\left(t_{g}, v, \alpha_{g}, \beta_{g}, h_{1}, h_{2 g}, h_{3 g}\right)=\sum_{g=1}^{G} \gamma_{g} A_{g} t_{g}^{\frac{1}{p}}+\nu\left(\mathbf{u}^{T} \mathbf{K} \mathbf{u}-\Delta+h_{1}^{2}\right)+ \\
& \sum_{g=1}^{G} \alpha_{g}\left(-t_{g}+t_{\min }+h_{2 g}^{2}\right)+\sum_{g=1}^{G} \beta_{g}\left(t_{g}-t_{\max }+h_{3 g}^{2}\right) .
\end{aligned}
$$

2.1.4. Kuhn-Tucker conditions. Neglecting the details, one can obtain

$$
\begin{array}{r}
\frac{\partial £}{\partial t_{g}}=\frac{1}{p} \gamma_{g} A_{g} t_{g}^{\frac{1-p}{p}}+\nu\left(\frac{\partial \mathbf{u}^{T}}{\partial t_{g}} \mathbf{K} \mathbf{u}+\mathbf{u}^{T} \frac{\partial \mathbf{K}}{\partial t_{g}} \mathbf{u}+\mathbf{u}^{T} \mathbf{K} \frac{\partial \mathbf{u}}{\partial t_{g}}\right)-\alpha_{g}+\beta_{g}=0 \\
(g=1, \ldots, G) .
\end{array}
$$

Due to symmetry of the stiffness matrix $\mathbf{K}$ and other simplifications equation (4) can be replaced by the following relation

$$
\frac{\partial £}{\partial t_{g}}=\frac{1}{\mathrm{p}} \gamma_{g} A_{g} t_{g}^{\frac{1-\mathrm{p}}{\mathrm{p}}}-\nu \sum_{e=1}^{E_{s}} u_{g e}^{T} \frac{\partial \mathrm{K}_{\mathrm{ge}}}{\partial t_{g}} u_{g e}-\alpha_{g}+\beta_{g}=0 ; \quad(g=1, \ldots, G),
$$

where the subscript ge refers to thee-th finite element of the $g$-th ground element. 
If the "normalized" element stiffness matrix is $\tilde{\mathbf{K}}_{g e}$ (e.g. calculated for a unit thickness $\left.\left(t_{g}=1\right)\right)$, then the element stiffness matrix $\mathbf{K}_{g e}$ for actual thickness $t_{g}$ is expressed by $\mathbf{K}_{g e}=t_{g} \tilde{\mathbf{K}}_{g e}$ due to the linear relation and $\frac{\partial \mathbf{K}_{g e}}{\partial t_{g}}=\tilde{\mathbf{K}}_{g e}$. Introducing the following notation $R_{g}=t_{g}^{2} \sum_{e=1}^{E_{g}} \mathbf{u}_{g e}^{T} \tilde{\mathbf{K}}_{g e} \mathbf{u}_{g e}$ equation $(5)$ becomes very simple

$$
\frac{1}{p} \gamma_{g} A_{g} t_{g}^{\frac{1-p}{p}}-\nu \frac{R_{g}}{t_{g}^{2}}-\alpha_{g}+\beta_{g}=0 .
$$

Continuing the derivations:

$$
\begin{array}{llrl}
\frac{\partial £}{\partial \nu}=\mathbf{u}^{\mathbf{T}} \mathbf{K} \mathbf{u}-\Delta+h_{1}^{2}=0 & \text { and } & \frac{\partial £}{\partial h_{1}}=2 \nu h_{1}=0, \\
\frac{\partial £}{\partial \alpha_{g}}=-t_{g}+t_{\min }+h_{2 g}^{2}=0 & \text { and } & \frac{\partial £}{\partial h_{2 g}}=2 \alpha_{g} h_{2 g}=0, \\
\frac{\partial £}{\partial \beta_{g}}=t_{g}-t_{\max }+h_{3 g}^{2}=0 & \text { and } & \frac{\partial £}{\partial h_{3 g}}=2 \alpha_{g} h_{3 g}=0 .
\end{array}
$$

Omitting the details from equations $(6 c),(7),(8)$ and $(9)$ the values of the Lagrange multipliers, slack variables and the thickness values $t_{g}$ can be calculated iteratively.

As it is in COC type methods, before the calculation of the Lagrange multiplier $\nu$, one needs to define a range for the thickness: a set of active and passive thicknesses.

There exist three possibilities:

If $t_{\min }<t_{g}<t_{\max }$ (or the ground element is "active", $g \in \mathcal{A}$ ) then $\alpha_{g}=\beta_{g}=0$ and by $6 \mathrm{c}$ the following formula can be obtained

$$
t_{g}=\left(\frac{\nu p R_{g}}{A_{g} \gamma_{g}}\right)^{\frac{p}{p+1}}
$$

In case of $t_{g}=t_{\min }$ the corresponding Lagrange multipliers are $\alpha_{g} \geqslant 0, h_{2 g}=0$ and (6c) implies

$$
t_{g} \geqslant\left(\frac{\nu p R_{g}}{A_{g} \gamma_{g}}\right)^{\frac{p}{p+1}}
$$

This means that if (10) gives a $t_{g}$ - value which is smaller than $t_{\min }$ then (6c)) is satisfied by $t_{g}=t_{\text {min }}$. Similarly, in case of $t_{g}=t_{\max }$ the corresponding Lagrange multipliers are $\beta_{g} \geqslant 0, h_{3 g}=0$ and then $6 \mathrm{c}$ implies

$$
t_{g} \leqslant\left(\frac{\nu p R_{g}}{A_{g} \gamma_{g}}\right)^{\frac{p}{p+1}}
$$

which allows $t_{g}=t_{\max }$ when 10 gives a $t_{g}$ - value which is greater than $t_{\max }$. If $t_{g}=t_{\min }$ or $t_{g}=t_{\max }$ we call the ground element "passive" $(g \in \mathcal{P})$.

2.1.5. Calculation of the final iterative formulas. In order to keep the number and layout of ground elements constant and avoid the ill-conditioned stiffness matrix, one can replace the zero element thickness $\left(t_{\text {min }}\right)$ with a small but finite value (e.g. 
$t_{\text {min }}=10^{-6}$ ). If the single displacement constraint is active in problem (4) (e.g. satisfies the equality sign) the following form holds

$$
\Delta-\sum_{g=1}^{G} \frac{R_{g}}{t_{g}}=0 .
$$

Since the thickness value for passive elements $(g \in \mathcal{P})$ is given and for active elements $(g \in \mathcal{A})$, it can be calculated from 10 that

$$
\Delta-\sum_{g \in \mathcal{P}}^{\frac{R_{g}}{t_{g}}}=\sum_{g \in \mathcal{A}}^{\frac{R_{g}}{t_{g}}}=\sum_{g \in \mathcal{A}} \frac{R_{g}}{\left(\frac{v p R_{g}}{A_{g} \gamma_{g}}\right)^{\frac{p}{p+1}}}
$$

implying

$$
\nu^{\frac{p}{p+1}}=\frac{\sum_{g \in \mathcal{A}}\left(\frac{A_{g} \gamma_{g}}{p}\right)^{\frac{p}{p+1}} R_{g}^{\frac{1}{p+1}}}{\Delta-\sum_{g \in \mathcal{P}}^{\frac{R_{g}}{t_{g}}}} \quad(\text { for } \mathcal{A} \neq 0) .
$$

The optimal solution can be obtained by evaluating iteratively the thickness values $t_{g}$ and the Lagrange-multiplier from $(10)$ and $(15)$.

2.1.6. The Applied SIMP Algorithm can be defined as follows:

1. Specify the Max and Min value of $t_{g},\left(t_{g \max }=1, t_{g \text { min }}=10^{-6}\right)$.

2. Specify a maximum displacement $\Delta$ (single displacement).

3. Set the penalty value, $p=1$, later this value will be incremented to $p=1.5,2$, etc.

4. Specify design domain, including supports and loading.

5. Carry out FEM.

6. Extract displacement field for entire structure $\mathbf{u}^{T}$.

7. Calculate $R_{g}$ with displacement vector based on current element solution set $t_{g}$, but using the stiffness matrix for the elements as if it had $t_{g}=1$.

8. Calculate Lagrange multiplier $\nu$ :

$$
\nu=\frac{\sum_{g \in \mathcal{A}}\left(\frac{A_{g} \gamma_{g}}{p}\right) R_{g}^{\frac{1}{p}}}{\left(\Delta-\sum_{g \in \mathcal{P}}^{\frac{R_{g}}{t_{g}}}\right)^{\frac{p+1}{p}}} \quad(\text { for } \mathcal{A} \neq 0) .
$$

9. Calculate new element solution set:

$$
t_{g, \text { new }}=\left(\frac{\nu p R_{g}}{A_{g} \gamma_{g}}\right)^{\frac{p}{p+1}}
$$

where $\nu^{\frac{p}{p+1}}$ is the Lagrange multiplier calculated in step 8 with the correct power. 
10. Determine set of active and passive elements by the following element limit set:

$$
\begin{array}{llll}
t_{g, \text { new }}=t_{\min } & \text { if } & t_{g, \text { new }} \leqslant t_{\min }=10^{-6} ; & e \in \mathcal{P}, \\
t_{g, \text { new }}=t_{\max } & \text { if } & t_{g, \text { new }} \geqslant t_{\max }=1 ; & e \in \mathcal{P}, \\
t_{\text {g,new }}=t_{\text {g,new }} & \text { if } & t_{\text {min }} \leqslant t_{g} \leqslant t_{\max }=1 ; & e \in \mathcal{A} .
\end{array}
$$

11. If active set has changed from the previous iteration, go to step 5 , else if active set has not changes from pervious iteration go to step 12 .

12. Increment $p$ until all the elements become passive or reach the limit for $p$, using following formula: $p=p$ +increment (step size is controlled).

In topology optimization the checker-board pattern -what is a numerical artifacts with artificially high stiffness is a big problem. To avoid it as an optimal solution there are several techniques (see e.g. Sigmund and Petersson [28]). Here a simple procedure was used which was published by Gáspár, Lógó and Rozvany [9]. The key point is that all the ground elements in case of disk (a primary meshing provides the so-called ground elements) should sub-divide into further finite elements (secondary elements). For the subdivision it is enough to use 2 by 2 elements. Further number of sub-elements can not improve significantly the final result.

\section{Numerical examples}

The 100 years old Michell's theory on least-weight trusses [13] provides an optimality criteria formula for a continuum in case of low volume fraction. Since his solution usually consists of an infinite number of members, Prager [16] termed Michell's frameworks "truss-like" structures. The Michell's theory was generalized to other structures by Prager and Rozvany (e.g. [17]). During this century several researcher added further result to this topic, but among them Rozvany's school made the most intensive research on it and published many papers with very significant results (e.g. Rozvany $[21,22,24,27])$. In this Chapter some new optimal topologies are introduced. The computer program is based on the above mentioned algorithm. 4-nodes quadrilateral and 2 nodes truss FE's are used. In each case the normalized "thickness" of the finite elements is used as design variable and a unit Young's Modulus is applied. The displacement limit is always $150 \%$ of initial displacement obtained from the initial design at the load location.

3.1. Example 1. "MBB beam". Consider a rectangular ground structure with hinge support in one end and roller support at the other end (Figure 3). The aspect ratio (height over length) at the initial design is 1 to 4 .

The total number of the finite elements is 9600 . The applied load is 100 unit at the middle of the top of the design domain. Only 4-nodes quadrilateral FE's are used The penalty parameter $p$ was run from $p=1$ to $p=3$ with smooth increasing (increment is 0.25 ). The Poisson ratio is 0. The exact analytical solution (e.g. Rozvany et al. [21] ) is shown in Figure 4. 


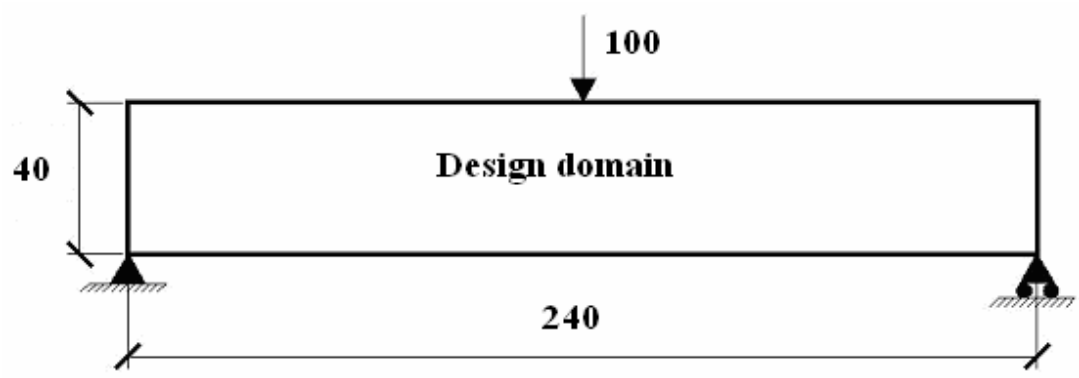

Figure 3. Design domain with boundary conditions

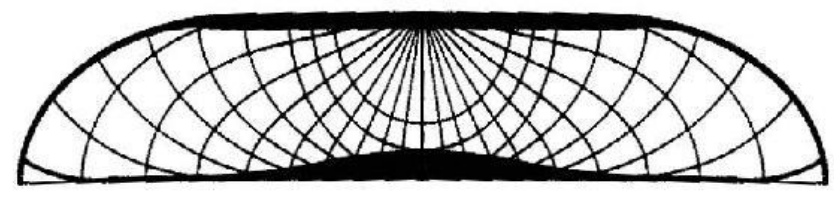

Figure 4. Exact analytical solution after Rozvany et al.

The solution in Figure 5 agrees closely with the exact analytical solution with zero volume fraction. One can see that by the use of $2 \times 24$-nodes FE's per ground element the checkerboard pattern is completely suppressed (Figure 2).

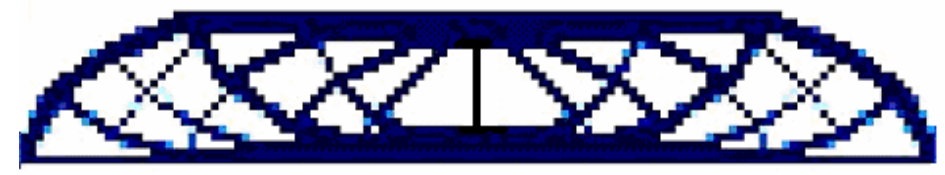

Figure 5. Optimal topology obtained by numerical calculation

3.2. Example 2. Rectangular domain with unequal point loads and unsymmetrical location. An intensively investigated test structure is rectangular domain with two hinge supports and two point loads [12,21,27]. The usual aspect (height/length) ratio is 0.5 and the supports are located at the middle of the left and right edges, respectively. Sometimes this ratio is not enough because the active zone reaches the top and the bottom edges of the design domain. To avoid this effect the height of the design domain is increased and the aspect ratio is 0.7 (Fig.4.).

In this example the effect of the unsymmetrical location and magnitude of the right hand point loading is investigated. The ratio of magnitudes of the unequal point loads is 2 (left load is 100 units, right load is 50 units). The left hand load is always located at $1 / 4$ of the horizontal dimension while the location of the right load is varied from 


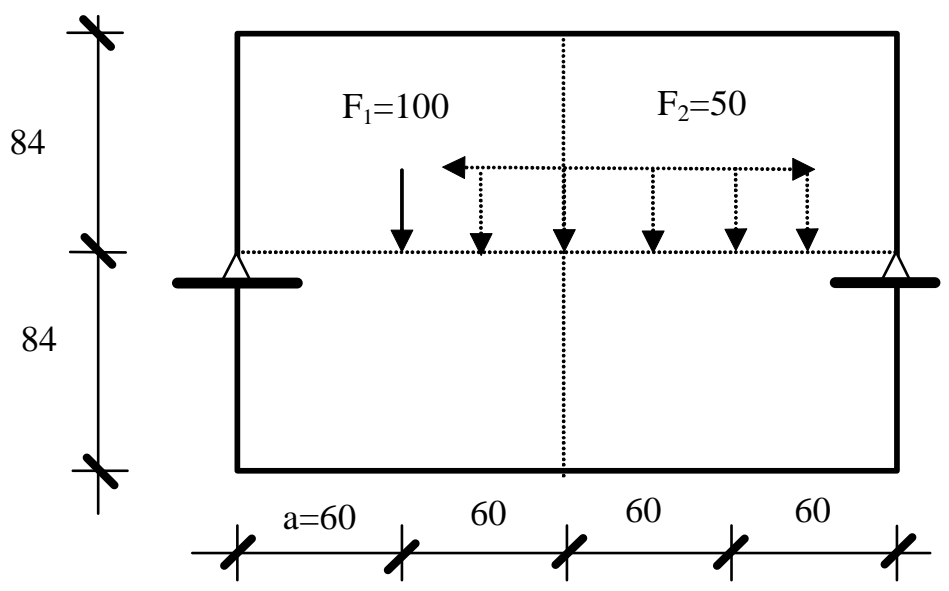

Figure 6. Design domain for variable location of $\mathbf{F}_{2}$

$2 / 8$ to $7 / 8$ of the horizontal dimension. Total number of finite elements is 40320 by the use of $84 \times 120$ ground elements and $2 \times 2$ sub-elements. The Poisson ratio is equal to 0 . The same type of penalty parameter $p$ was applied (from $p=1$ to $p=1.5$ with smooth increasing (increment is 0.1 ) and later to $p=3.5$ with increment $=0.25$ ).

The pictures of the optimal topologies in function of the right force location can be found in Table 1. The exact analytical solution for zero volume fraction can be seen in Fig.5. after Melchers [12]. The obtained numerical solutions are in good agreement with the analytical solutions.

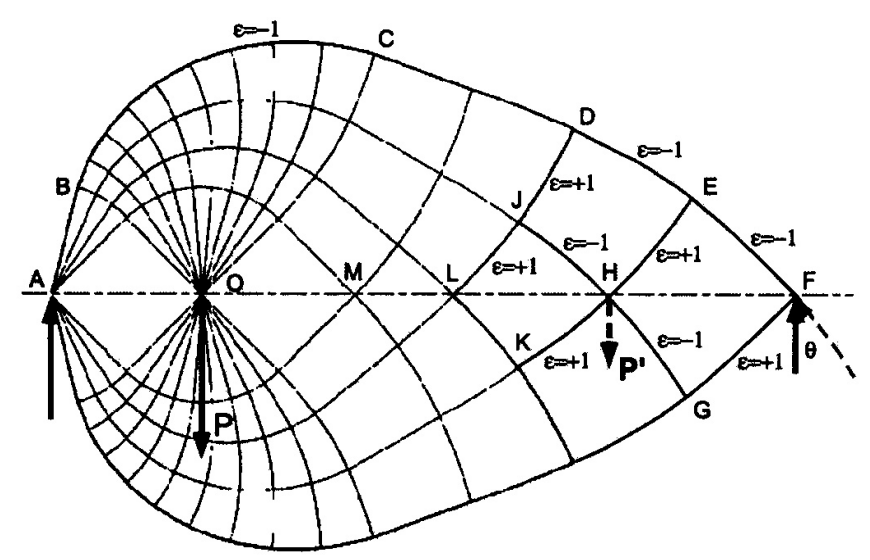

Figure 7. Exact analytical solution after Melchers. 
Table 1. Optimal topologies for unsymmetrical loading conditions

$\left.\begin{array}{c}\text { Locat } \\ \text { ion of } \\ F_{2}\end{array}\right)$
Optimal Solution $\begin{gathered}\text { Locat } \\ \text { ion of } \\ F_{2}\end{gathered}$

To demonstrate the evolution of the optimal topologies during the major iterations the intermediate results (correspond to different penalties) are displayed. One can see in Figure that enough a few major iterations to reach the final topologies which proves the robustness of the applied algorithm. At the beginning there are intermediate thicknesses (light colours), what disappears at the end of the program (only two thicknesses exist: 1or 0 ) . 


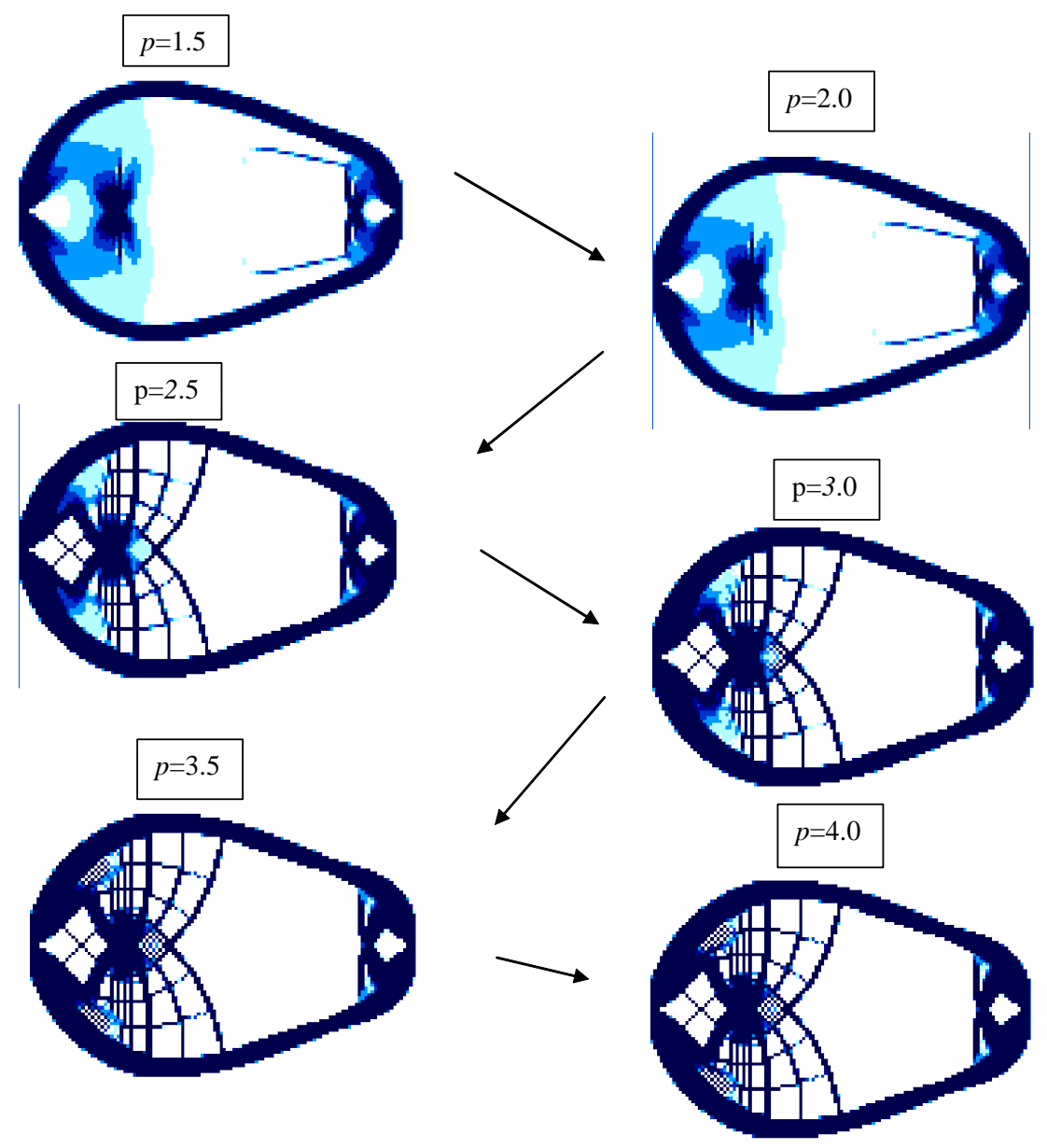

Figure 8. Evolution of the optimal topology in function of the penalty parameter

3.3. Example 3. Topology optimization for variable external forces of nonzero cost. Classical theories of variable force (mostly support) optimization, based on optimality criteria and adjoint displacement fields, were developed in the mid-seventies (see e.g. Rozvany and Mroz [19]. More recently, Buhl [7] and Pomezanski [14], Rozvany et al. [26] ) developed numerical methods for topology optimization with allowance for the cost of supports. This example discusses the problem of optimizing structural topologies when some of the external forces are variable and they have a nonzero cost. Such forces may represent a reaction at a support, a force generated by passive control or a ballast (weight) used for increasing cantilever action or modifying natural frequencies. In the presented examples, it is assumed that the cost 
of external forces depends on their magnitude and in this way it is proportional to the volume of the optimal topology.

Assuming the same permissible stress in tension and compression, in the objective function of optimization problem (4) the formulation for bars can be reformulate as follows:

$$
\min \left(\sum_{i} k \ell_{i}\left|F_{i}\right| t_{i}^{\frac{1}{p}}+\sum_{j} b \ell_{j}\left|R_{j}\right| t_{j}^{\frac{1}{p}}\right)
$$

where $k$ and $b$ are given constants, $F_{i}$ are bar forces and $R_{j}$ are reaction forces. Replace all supports with a set of bars, such that in the direction of any potential reaction force. (At roller support, for example, we have only one fictitious bar and at a pin support, we have an infinite number of fictitious bars in all possible directions.) It is easy to proof that the optimality conditions become the same as was indicated before.

Let's consider a rectangular design domain with a single point load at middle of the right edge (Figure 10.) On the left side there are fix hinges at the top and the bottom corners and there are pin supports at the $1 / 4$ and $3 / 4$ of the left side, respectively. (Here the "infinite number" of bars means at the pin support that initially there is a

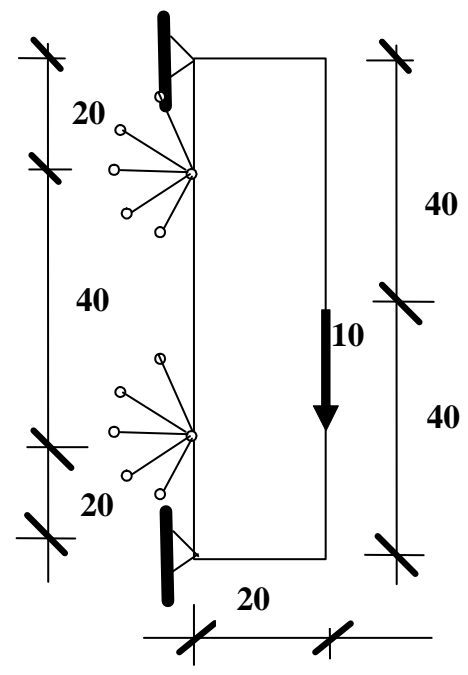

Figure 9. Design domain for variable supports

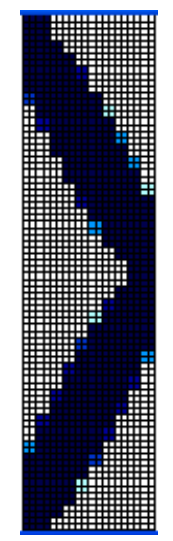

Figure 10. Optimal topology in Case A.

bar in every five degree direction.) The cost of the supports are varied from 1 (no cost) to 10000 (expensive). To demonstrate the method and the algorithm, three cases are presented: Case A: the bars have high cost while the hinges have no cost; Case B: all supports have cost, but the bars have three times more cost than the hinges, Case C: all the supports have same unit cost. For the continuum design domain 4-nodes quadrilateral FE's are used Total number of finite elements is 1600 by the use of $10 \times 40$ ground elements and $2 \times 2$ sub-elements. The Poisson ration is 0 . 
Same type of penalty parameter $\mathrm{p}$ was applied (from $p=1$ to $p=1.5$ with smooth increasing (increment is 0.1 ) and later to $p=4.0$ with increment=0.25). The numerical results can be seen in Figures 10-11. They are in good agreement with the expected ones. In Figures 12-13. among the bar supports only one member at the bottom and one member at above are active. Their directions are 135 and 225 degree, respectively.

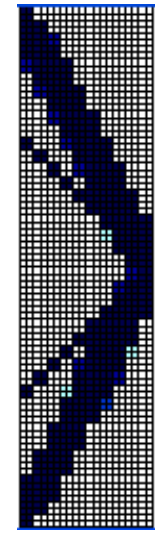

Figure 11. Optimal topology in Case B.

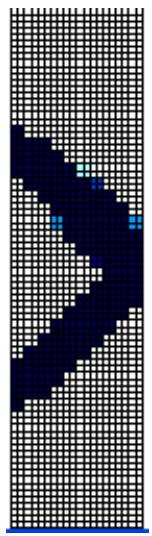

Figure 12. Optimal topology in Case C.

\section{Conclusion}

A very efficient iterative algorithm was presented for topology design of continuum type structures by the use of displacement constraint. The derived algorithm is suitable for support design as well. The applied meshing provides a good technique to avoid the checkerboard pattern. By the use of the smooth penalization increment the obtained numerical solutions are in good agreement with the existing and proved analytical solutions. Conceptually this topology design is simple, since the algorithm does not require intensive mathematics. The number of the design variables (thousands) significantly exceeds the maximum number of variables what can be used in any kind of mathematical programming algorithm. The main disadvantage is that the buckling and other constraints are not taken into consideration during the optimization procedure but the obtained numerical topologies are a good starting points for further optimal design. The support optimization technique is suitable to demonstrate the effects of strengthening of structures.

Acknowledgement. The present study was supported by the Hungarian National Scientific and Research Foundation (OTKA) (grant T 037922) and the Bolyai Janos Scholarship.

\section{References}

1. Allaire, G.; Kohn, R.: Topology Optimization and Optimal Shape Design Using Homogenization. In: Bendsoe, M.P.; Mota Soares, C.A. (eds.) Topology Design of Structures (Proc. NATO ARW, Sesimbra, Portugal 1992), Dordrecht: Kluwer, (1993), 207218. 
2. Barta, J.: On the minimum weight of certain redundant structures, Acta Tech. Acad. Sci. Hung., V.18, (1957), 67-76.

3. Bendsoe, M. P. and Kikuchi, N.: Generating optimal topologies in structural design using a homogenization method. Comp. Meth. Appl. Mech. Eng. 71, (1988), 197-224.

4. Bendsoe, M.P. and Mota Soares, C. A.: Topology Design of Structures (Proc. NATO ARW, Sesimbra, Portugal, 1992), Dordrecht, Kluwer, 1993.

5. Bendsoe, M. P. and Sigmund, O.: Topology Optimization: Theory, Methods and Applications. Berlin: Springer, 2003.

6. Berke, L.: An Efficient Approach to the Minimum Weight Design of Deflection Limited Design, AFFDL-TM-70-4-FDTR, (1970), Wright-Patterson AFB.

7. Buhl, T.: Simultaneous topology optimization of structure and supports. Struct. Multidisc. Optim., 23, (2002), 336-346.

8. Cheng, K. T. and Olhoff, N.: An investigation concerning optimal design of solid elastic plates. Int. J. Solids Struct., 17, (1981), 305-323.

9. GÁspár, Zs., Lógó, J. and Rozvany, G. I. N.: On design-dependent constraints and singular topologies. Struct. and Multidisc. Opt., 24(4), (2002), 338-342.

10. KALiszKY, S. and LóGó, J.: Optimal design of dynamically loaded reinforced concrete frames under displacement and rotation constraints. Journal of Structural Optimization, 3, (1991), 121-131.

11. Lógó, J. and Ghaemi, M.: Topology optimization by SIMP method. Proc. of the Conference on Numerical Methods and Computational Mechanics, Miskolc, Hungary, July 15-19, (2002), 165-166.

12. Melchers, R. E.: On extending the range of michell-like optimal topologies structures. Structural and Multidisc. Optimization, 29(2), (2005), 85-92.

13. Michell, A. G. M.: The limits of economy of material in frames structures. Phil. Mag., 8, (1904), 589-597.

14. Pomezanski, V.: Changing the connections of structural elements during an optimization process, Journal of Computational and Applied Mechanics, 5(1),(2004), 117-127.

15. Prager, W. and Taylor, J. E.: Problems of optimal structural design. J. Appl. Mech. ASME, 35, (1968), 122-106.

16. Prager, W.: Introduction to Structural Optimization. CISM Courses and Lectures Notes 212, Springer Verlag, Wien-New York, 1974.

17. Prager, W. and Rozvany, G. I. N.: Optimal layout of grillages. J. Struct. Mech., 5, (1977), 1-18.

18. Rossow, M. P. and TaYlor, J. E.: A finite element method for the optimal design of variable thickness sheets. J. AIAA, 11, (1973), 1566-1569.

19. Rozvany, G. I. N. and Mroz, Z.: Column design: Optimization of support conditions and segmentation. J. Struct. Mech., 5, (1977), 279-290.

20. Rozvany, G. I. N.: Structural Design via Optimality Criteria. Kluwer Academic Publisher, Dordrect, 1989.

21. Rozvany, G. I. N., Bendsoe, M. P. and Kirsh U.: Layout optimization of structures. Appl. Mech. Reviews., 48(2), (1995), 41-118. 
22. Rozvany, G. I. N.: Some shortcomings in Michell's truss theory, Struct. Optim., 12, (1996) 244-250.

23. Rozvany, G. I. N.: Optimization in Structural Mechanics. CISM Courses and Lectures Notes 374, Springer Verlag, Wien-New York, 1997.

24. Rozvany, G. I. N., Gollub, W. and Zhou, M.: Exact Michell trusses for various combinations of line supports. Part II, Struct. Optim., 14, (1997), 138-149.

25. Rozvany, G. I. N.: stress ratio and compliance based methods in topology optimization - a critical review. Structural and Multidisc. Optimization, 21, (2001), 109-119.

26. Rozvany, G. I. N., Lógó, J. and Kaliszky, S.: Topology Optimization for Variable External Forces of Nonzero Cost. Proc. of $5^{\text {th }}$ World Congress of Structural and Multidisciplinary ptimization, May 19-23, 2003, Lido di Jesolo, Venice, Italy. 323-324.

27. Rozvany, G. I. N., LóGó, J. and Querin, O. M.: New Classes of Analytically Derived Optimal Topologies and Their Numerical Confirmation, XXI International Congress of Theoretical and Applied Mechanics, Warsaw, Poland, August 15-21, 2004.

28. Sigmund, O. and Petersson, J.: Numerical Instabilities in Topology Optimization: A Survey on Procedures Dealing with Checkerboards, Mesh-dependencies and Local Minima. Struct. Optim. 16, (1998), 68-75.

29. TAYlor, J. E.: Strongest column: an energy approach. J. Appl. Mech., 89(2), (1967), 486-487.

30. Zhou M. and Rozvany, G. I. N.: The COC Algorithm, Part II: Topological, Geometrical and Generalized Shape Optimization. Comp. Meth. Appl. Mech. Eng. 89, (1991), 309-336. 